\title{
Fatores de risco adicionais à Escala de Braden: um risco para úlceras de pressão
}

Recebido em: 12/04/2011

Aceito em: 16/11/2011

\author{
Marisa Hans ${ }^{1}$ \\ Júlia Valéria de Oliveira Vargas Bitencourt ${ }^{2}$ \\ Flaviana Pinheiro ${ }^{3}$
}

Estudo quantitativo que tem por objetivo analisar a presença de fatores de risco de úlceras de pressão (UPs), adicionais à Braden, com 134 clientes internados no CTI do Hospital Mãe de Deus, em maio/ junho de 2010 com aprovação dos comitês institucionais: 39/2010; 353/10. Realizou-se coleta de informações do prontuário dos clientes, com instrumento estruturado. Dos 134 clientes, 43 desenvolveram UP, com fator de risco adicional à Braden: infecção, sepse, corticoides, noradrenalina, ventilação mecânica, edema, diabetes mellitus, insuficiência respiratória aguda, doenças inflamatórias, respectivamente com um $p<0,001$ e neoplasias e doenças imunossupressoras com $\mathrm{p}<0,05$, assim, com significância estatística. Entretanto, sem possibilidade de comparação significativa, considerando o reduzido quantitativo de estudos, tratando da problemática. Portanto, na avaliação do risco das UPs devem ser agregados outros fatores, visando a otimização das medidas de prevenção e qualificação assistencial, além de haver mais estudos permitindo a comparação.

Descritores: Úlcera de Pressão, Escala de Braden, Prevenção.

\section{Additional risk factors related to Braden Scale: a risk for pressure ulcers}

This is a quantitative study that aims to analyze the presence of risk factors for pressure ulcers (UPs), in addition to Braden, with 134 clients admitted to the ICU of the Mother of God Hospital in May / June 2010 with the approval of institutional committees: 39 / 2010, 353/10. We carried out data collection from medical records of clients with structured instrument. Of the 134 clients, 43 developed UP, with the additional risk factor Braden: infection, sepsis, corticosteroids, noradrenaline, mechanical ventilation, edema, diabetes mellitus, acute respiratory failure, inflammatory diseases, respectively with $p<0.001$ and immunosuppressive diseases and cancers $p<0.05$, thus statistically significant. However, without the possibility of meaningful comparison, considering the small quantity of studies, dealing with the problem. Therefore, the risk assessment of UPs must be added other factors in optimizing the prevention and care skills, and be more studies allowing the comparison. Descriptors: Pressure Ulcers, Braden Scale, Prevention.

\section{Factores de riesgo adicionales a la escala Braden: un riesgo para las úlceras por presión}

Se trata de um estudio cuantitativo que tiene por objectivo analizar la presencia de factores de riesgo de úlceras por presión (UP), además de Braden, con 134 pacientes admitidos a la UCI del Hospital de la Madre de Dios en mayo / junio de 2010 con la aprobación de los comités institucionales: 39 / 2010, 353/10. Se llevó a cabo la recopilación de datos de los registros médicos de los clientes con instrumentos estructurados. De los 134 clientes, 43 desarrollaron UP, con la Braden factores de riesgo adicionales: infección, sepsis, los corticosteroides, la noradrenalina, la ventilación mecánica, edema, diabetes mellitus, insuficiencia respiratoria aguda, enfermedades inflamatorias, respectivamente, con $p$ enfermedades $<0,001$ e inmunosupresores y cánceres $p<0,05$, por lo tanto estadísticamente significativa. Sin embargo, sin la posibilidad de comparación significativa, considerando la pequeña cantidad de estudios, para tratar el problema. Por lo tanto, la evaluación del riesgo de UPS hay que añadir otros factores en la optimización de la prevención y técnicas de atención y ser más estudios que permitan la comparación.

Descriptores: Las Úlceras por Presión, Escala de Braden, La Prevención.

\section{INTRODUÇÃO}

A mobilização da enfermagem no sentido de implementar práticas efetivas em relação à prevenção ao desenvolvimento de úlceras de pressão (UPs) remete à Idécada de 1970, em que essa intenção fez com que vários pesquisadores elaborassem escalas para predizerem o risco. Destas, as mais citadas são a de Norton, construída em 1970, de Gosnell, em 1973, Waterlow, em 1979, e a mais atual, a de Braden, de 1987(1).

A escala de Braden vem sendo, consistentemente, utilizada nos EUA e no Brasil para tanto. Paranhos e Santos, em 1999, a validaram, sendo assim, traduzida para a língua portuguesa, adaptada e testada ${ }^{(2)}$. Desta forma, destacam-se a seguir os aspectos da escala.

Ela avalia seis fatores de risco no paciente, entre eles: Percepção Sensorial, Umidade, Atividade, Mobilidade, Nutrição, Fricção e
Cisalhamento. Ao fim da avaliação, chegamos a uma pontuação, que nos diz: Abaixo de 12=Risco elevado, 13-14 = Risco moderado, $15-16=$ Baixo risco $^{(1: 8)}$.

Neste sentido, a unidade hospitalar que mais tem chance de apropriação da escala é o Centro de Terapia Intensiva (CTI), já que os clientes apresentam-se em estado grave, instáveis hemodinamicamente, apresentando descompensação dos sistemas orgânicos com potencial de risco acentuado para o desenvolvimento de UPs. Logo, reforça-se que os clientes do CTI desenvolvem risco para UP, devido às suas limitações físicas, emocionais, com alteração do nível de consciência por estarem muitas vezes sedados, em suporte ventilatório, usando drogas vasoativas, com diversas situações de mal-estar, acamados por um

1 Enfermeira. Graduada no Curso de Enfermagem do IPA. 
longo período de tempo, dentre outros fatores ${ }^{(3,4)}$.

Entretanto, observa-se que mesmo com a utilização da Braden, e a consequente implementação dos cuidados diários, a incidência de UPs ainda é expressiva. Reflete-se que existam fatores de risco adicionais, não previstos na mesma, fazendo parte muitas vezes da condição intrínseca do cliente de CTI.

Nessa perspectiva, configura-se a dificuldade na detecção desses fatores, por carência de instrumentos sinalizadores, ocasionando incapacidade profissional em identificar as reais necessidades de cuidados que minimizem esta problemática. Assim, além da implicação direta ao cliente, envolvendo a qualidade assistencial, sobrepõe-se a elevação do custo quanto aos cuidados a este cliente com UP, secundário ao aumento do tempo de hospitalização, uso de materiais extras e profissionais habilitados, exigindo mais horas de cuidados de enfermagem. Diante disso, o objetivo deste estudo foi o de analisar a presença de fatores de risco ao desenvolvimento de UP, que sejam adicionais à Escala de Braden, em clientes internados no CTI do Hospital Mãe de Deus (HMD).

\section{METODOLOGIA}

Estudo quantitativo realizado no CTI do HMD em Porto Alegre/ RS, com investigação, através de formulário, em prontuários de 134 clientes com idade superior a 18 anos, internados no período de maio a junho de 2010, após aprovação dos Comitês do Centro Universitário Metodista do Sul - IPA e da instituição concedente, cujos protocolos são: 39/2010;353/10. Os dados foram analisados no programa Statistical Package for Social Science (SPSS) versão 17.0 Windows, e após tratamento estatístico, obtiveram-se tabelas e gráficos.

\section{APRESENTAÇÃO DOS RESULTADOS}

As tabelas a seguir evidenciam o perfil dos clientes do CTI do HMD, destacando-se que a idade média foi de 68,5 anos.

Assim, 60\% eram do sexo masculino, cujos diagnósticos mais prevalentes foram: sepse (16\%), infarto agudo do miocárdio (IAM) (12\%), insuficiência respiratória aguda (IRA) e acidente vascular cerebral (ACV) com, respectivamente, 11\% cada um.

As situações clínicas preexistentes mais prevalentes, consideradas comorbidades, foram: IRA (53\%); Insuficiência Cardíaca Congestiva (ICC) (52\%) e Diabetes Mellitus (DM) (49\%).

Adiante, visualizam-se os agravos pós-internação, assim como implementações terapêuticas com potencial iatrogênico. Nesse sentido, as variáveis consideradas foram: infecção e edema como agravos e ventilação mecânica (VM), uso de corticoides e de noradrenalina como elementos terapêuticos.

Dessa forma, 49\% dos clientes desenvolveram edema corporal e 39\% tinham alguma infecção associada ao quadro.

Na tabela 4, salientamos o uso da VM em 63\% dos clientes, a utilização de corticoides em 57\%, e da noradrenalina em $48 \%$ dos clientes.

No desfecho da apresentação dos resultados, evidenciam-se os dados de maior relevância para este estudo, uma vez que elucidam estatisticamente qual a significância da correlação do desenvolvimento de UP com os fatores de risco adicionais à Escala de Braden. Sendo assim, os dados respondem ao objeto desta pesquisa. Faz-se a ressalva de que as variáveis analisadas para significância correspondem àquelas até então visualizadas nas tabelas acima. Acrescenta-se que, para a correlação, avaliou-se o desenvolvimento de úlceras de pressão (UPs) na CTI do HMD após admissão nesta unidade.

Percebeu-se que dos $100 \%$ (134) de clientes, 32,1\%(4), desenvolveram UPs, e $67,9 \%$ não desenvolveram no período da internação. $\mathrm{E}$, quanto à significância atribuída a cada uma das variáveis analisadas como adicionais à escala de Braden ao desenvolvimento de UP, o estudo aponta: infecção, corticoides, sepse, noradrenalina, VM, edema, DM, IRA, doenças inflamatórias, respectivamente com um $\mathrm{p}<0,001$ e as variáveis neoplasias e doenças imunossupressoras com $p<0,05$. Dessa forma, todas com significância estatística e as variáveis sexo, ICC e insuficiência renal crônica (IRC) com p > 0,05 sem significância estatística.

\section{DISCUSSÃO}

No que tange ao perfil dos clientes internados na CTI do HMD, quando a média de idade foi de 68 anos, predominando o sexo masculino $80 \%$, e na correlação destas variáveis ao desenvolvimento de UP, não obtiveram significância, portanto, não configuram como fatores de risco adicionais à escala de Braden.

Ressalta-se que estudos nacionais apontam a idade como um dos fatores mais relevantes à fisiopatogênese das UPs, destacando-se que o predomínio da idade em um dos estudos foi acima de 60 anos (78,6\%), e o mesmo foi implementado em variadas unidades de uma instituição hospitalar ${ }^{(5)}$.

Em estudos desenvolvidos em CTI, concluiu-se que os clientes com UP tinham em média 67 anos, o que corresponde aproximadamente à média de idade da realidade pesquisada. Também, de forma similar, nestes estudos a idade não se apresentou como fator relevante ao desenvolvimento de $\operatorname{UP}^{(6,7)}$.

Quanto ao sexo, prevaleceu o masculino, sendo (60\% vs $53 \%$, $p=0,313)$, uma estatística não significativa para o desenvolvimento

Tabela 1 - Sexo e Motivo da Internação

\begin{tabular}{|l|l|l|}
\hline VARIAVEIS & N & $\%$ \\
\hline Sexo masculino & 80 & 60 \\
\hline Sepse & 21 & 16 \\
\hline Infarto Agudo do Miocárdio & 16 & 12 \\
\hline Insuficiência Respiratória Aguda & 15 & 11 \\
\hline Acidente Vascular Cerebral & 15 & 11 \\
\hline Doenças Abdominais & 13 & 10 \\
\hline Neoplasias & 9 & 7 \\
\hline Doenças Neurológicas & 9 & 7 \\
\hline Cirurgia Revascularização do Miocárdio & 7 & 5 \\
\hline Traumatismos & 7 & 5 \\
\hline Insuficiência Cardíaca Congestiva & 5 & 4 \\
\hline Parada Cardiorrespiratória & 5 & 4 \\
\hline Traumatismo Cranioencefálico & 3 & 2 \\
\hline Aneurismas & 3 & 2 \\
\hline Outros* & 4 & 4 \\
\hline
\end{tabular}

*Embolias, Edema Agudo de Pulmão (EAP), Fibrilação Atrial Crônica (ACFA) e Transplante de Rim. 
de UP. Nesse sentido, observou-se maior ocorrência de UP em clientes do sexo masculino, em um estudo no qual, inclusive, foi possível estabelecer significância (70\% vs 30\%, p=0,026(4). Por outro lado, em discordância a essa evidência, e ratificando a presente pesquisa, destaca-se um estudo no qual se indica não haver associação significantequanto ao desenvolvimento de UP e o sexo do cliente ${ }^{(8)}$.

Quanto à infecção, caracterizada por ser um agravo pós-internação, observou-se que a maioria dos clientes desenvolveu UP ( $81 \%$ vs $19 \%$ p<0,001), sendo essa diferença significativa.

A infecção diminui as atividades dos fibroblastos na produção de colágeno e estimula os leucócitos a liberar lisozimas, uma substância que destrói o colágeno, enfraquecendo a pele e as feridas. Esses microrganismos invasores capturam os nutrientes e o oxigênio necessários para a cicatrização, aumentando o risco para $\mathrm{UPs}^{(8)}$. A sepse, que consiste na resposta sistêmica à infecção grave, configurou-se como importante fator de risco ( $46 \%$ vs $10 \%, p<0,001)$.

Ela desestrutura toda a cadeia inflamatória e da coagulação, volume sanguíneo circulante, débito cardíaco, causa hipotensão e perfusão tecidual, causando hipoxemia ${ }^{(9)}$. Em meio a este quadro clínico, o cliente torna-se, compreensivelmente, mais suscetível à UP. Neste cenário de infecção e sepse, sobrepõese uma condição clínica grave, pela qual se impõe a adoção de associações terapêuticas como VM, vasopressores tipo noradrenalina e corticoides. Essas terapêuticas usadas na CTI no paciente grave constituem fatores de risco ao desenvolvimento de UP(10), e tiveram relevância na pesquisa VM ( $93 \%$ vs $48 \%$, $p<0,001$ ), noradrenalina ( $84 \%$ vs $31 \%, p<0,001$ ) e corticoides (79\% vs $47 \%, p<0,001)$.

Em específico indica-se que estes fármacos são responsáveis por aumentar o catabolismo proteico, inibir a síntese da proteína, afetar os mecanismos de defesa do corpo e inibir a produção de colágeno, aumentando a suscetibilidade a infecções, prejudicando a cicatrização, afinamento da pele e formando edema ${ }^{(11)}$. Logo, deixando os tecidos mais suscetíveis à UP.

A noradrenalina, uma catecolamina endógena secretada pela medula suprarrenal em resposta a estresse orgânico, com o objetivo de redistribuir o fluxo sanguíneo corporal, de tal forma a preservar órgãos nobres, aumentar a atividade cardíaca, traz como consequência uma redução na perfusão em leitos periféricos, potencializando a desnutrição tecidual da pele ${ }^{(12)}$.

Para a VM, é coerente afirmar que clientes com essa implementação terapêutica têm maior risco para desenvolver infecções ${ }^{(13)}$. Além disso, geralmente são sedados, mantendo um perfil limitado de mobilização corporal, e com aumento da pressão tecidual contra as proeminências ósseas.

Nesse contexto, no estudo de Costa, a VM (75\% vs 27\%, p 0,001), foi descrito como um fator de risco importante para predizer o aparecimento de UP. Ele observou que os clientes em VM, com predominância da respiração controlada, são mais suscetíveis, provavelmente por serem mais graves ${ }^{(8)}$.

O edema que é observado pelo excesso de líquido intersticial, podendo ocasionar oclusão capilar e isquemia tissular, se a pressão não for aliviada, predispondo à hipoxia, à morte celular e à inflamação tissular, apareceu com relevância de (95\% vs $21 \%$, $\mathrm{p}<0,001)$. Nesse sentido, é citado que o edema se desenvolve, pois os tecidos diminuem o consumo de oxigênio e nutrientes e os restos metabólicos são acumulados, fazendo com que o líquido seja transferido para o espaço intersticial. Este, por sua vez, diminui o fluxo sanguíneo e o metabolismo local, interferindo na oxigenação e na nutrição dos tecidos ${ }^{(14,15)}$. Resultados semelhantes a este também já foram descritos. Assim, em um estudo observou-se que (60\% vs $30 \%$, p 0,056) dos clientes com edema desenvolveram UP(7).

A DM cria condições favoráveis para o aparecimento de UP, havendo uma correlação significante de $(72 \%$ vs $37 \%$, $p<0,001)$. Trata-se de doença crônica que causa alterações em pequenos vasos que dificultam a perfusão tecidual(16). Existindo a probabilidade de ocorrer a diminuição da síntese de colágeno e da angiogênese, além da presença de arteriosclerose nos pequenos vasos, contribui para a redução do fornecimento de oxigênio tecidual, deixando a pele mais fina, ressecada, mais suscetível a rupturas e ao desenvolvimento de UP(17,18).

Complicações de saúde ou comorbidades observadas no cenário de pesquisa tiveram relevância, tais como: IRA com (86\% vs $37 \%, p<0,001)$, doenças inflamatórias ( $46 \%$ vs $9 \%, p<0,001)$, neoplasias (33\% vs $13 \%$ p 0,008) e doenças imunossupressoras (7\% vs $0 \%$, p 0,031).

No estudo de Fernandes ${ }^{(7)}$, evidenciou-se que as doenças respiratórias (42,3\%), cardíacas (11,5\%) e metabólicas (7,7\%), como a DM, foram mais frequentes nos clientes que desenvolveram UP.

Costa $^{(8)}$ também evidenciou que $85 \%$ dos diagnósticos de internação dos clientes críticos na CTI apresentaram disfunções neurológicas, neoplasias e DM, seguidos de cardiopatias e doenças respiratórias, patologias que promovem instabilidade hemodinâmica e limitam a mobilidade do cliente, exigindo muitas vezes repouso absoluto no leito e predispondo a UP. Assim, havendo discordância com este estudo apenas para doenças cardíacas como a ICC, pouco relevante (46\% vs $55 \%$, p 0,362). 
Finalizando, observou-se que clientes com IRC apresentaram-se sem relevância ( $30 \%$ vs $18 \%$, p 0,170). Porém, mesmo assim, salienta-se que a condição da IRC, devido à uremia, desnutrição, anemia e outros comprometimentos sistêmicos, desencadeia alterações tegumentares, tais como prurido intenso, pele mais seca e escamosa, consequentemente mais fina e sensível(9).

\section{CONSIDERAÇÕES FINAIS}

A escala de Braden é adotada como instrumento para avaliação do risco ao desenvolvimento de UP. No entanto, a este estudo acrescentaram-se alguns fatores de risco adicionais à escala de Braden como infecção, sepse, administração de corticoides e de noradrenalina, VM, edema, DM, IRA, doenças inflamatórias, neoplasias e doenças imunossupressoras todos analisados com extrema significância, do ponto de vista estatístico. Entretanto, a comparação desses resultados com outras pesquisas foi prejudicada, dada a limitação de publicações nacionais no que concerne a essa temática.

Enfatiza-se pois que a associação dos fatores de risco evidenciados neste estudo denotam a relevância em buscar mais conhecimento sobre o assunto, remetendo à reflexão
Tabela 5 - Significância da correlação do desenvolvimento de UP com os fatores de risco adicionais à Escala de Braden

\begin{tabular}{|l|c|c|c|c|}
\hline \multicolumn{2}{|c|}{ TODOS } & UP INTRA- & SEM UP \\
& $(\mathrm{N}=134)$ & $(\mathrm{N}=43)$ & $(\mathrm{N}=91)$ & P \\
\hline Idade, a mediana (P25-P75)* & $68,5(56-78)$ & $73(54-81)$ & $64,9(56-77)$ & 0,259 \\
\hline Homens (\%) & $80(59,7)$ & $23(53,5)$ & $57(62,2)$ & 0,313 \\
\hline Infecção & $52(38,8)$ & $35(81,4)$ & $17(18,7)$ & $<0,001$ \\
\hline Sepse & $29(21,6)$ & $20(46,5)$ & $9(3,9)$ & $<0,001$ \\
\hline Corticoides & $77(57,5)$ & $34(79,1)$ & $43(47,3)$ & $<0,001$ \\
\hline Nora & $64(47,8)$ & $36(83,7)$ & $28(30,8)$ & $<0,001$ \\
\hline VM & $84(62,7)$ & $40(93)$ & $44(48,4)$ & $<0.001$ \\
\hline Edema & $60(44,8)$ & $41(95,3)$ & $19(20,9)$ & $<0,001$ \\
\hline DM & $65(48,5)$ & $31(72,1)$ & $34(37,4)$ & $<0,001$ \\
\hline IRA & $71(53,0)$ & $37(86,0)$ & $34(37,4)$ & $<0,001$ \\
\hline Doenças Inflamatórias & $28(20,9)$ & $20(46,5)$ & $8(8,8)$ & $<0,001$ \\
\hline Neoplasias & $26(19,4)$ & $14(32.6)$ & $12(13,2)$ & 0,008 \\
\hline Doenças Imunossupressoras & $3(2,2)$ & $3(7)$ & $0(0)$ & 0,031 \\
\hline ICC & $70(52,2)$ & $20(46,5)$ & $50(54,9)$ & 0,362 \\
\hline IRC & $28(20,9)$ & $12(27,9)$ & $16(17,6)$ & 0,170 \\
\hline
\end{tabular}

*Percentil 25 e Percentil 75.

\section{Referências}

1. Lobosco F. O enfermeiro atuando na prevenção das úlceras de pressão. Rev Eletrônica Quadrimestr Enferm. 2008;13:1-15.

2. Paranhos WY, Santos VLCG. Avaliação de risco para úlceras de pressão por meio da escala de Braden, na língua portuguesa. Rev Esc Enferm USP. 1999;33(N. Esp.):191-204,

3. Souza CAC, Santos I, Silva LD. Apropriação de concepções de Neuman e Braden na prevenção de úlceras de pressão. Rev Enferm UERJ. 2004;12:280-5. 4. Fernandes LM, Caliri MHL, Haas VJ. Efeito de intervençōes educativas no conhecimento dos profissionais de enfermagem sobre prevenção de úlceras pressão. Acta Paul Enferm. 2008;21(2):305-11.

5. Rogenski NMB, Santos VLCG. Estudos sobre a incidência de úlceras por pressão em um hospital universitário. Rev Latinoam Enferm. 2005;13(4):474-80.

6. Petrolino HMBS. Úlceras de pressão em pacientes de UTI: incidência, avaliação de risco e medida de prevenção [dissertação]. São Paulo: Escola de Enfermagem da Universidade de São Paulo; 2002.

7. Fernandes NCS. Úlceras de pressão: um estudo com pacientes de unidade de terapia intensiva [dissertaçäo]. Natal: Departamento de Enfermagem da Universidade Federal do Rio Grande do Norte; 2005.

8. Costa IG. Incidência de úlceras de pressão e fatores de risco relacionados em pacientes de um Centro de Terapia Intensiva [dissertação]. São Paulo: Escola de Enfermagem de Ribeirão Preto da USP; 2003. sobre a utilização da Escala de Braden e uma forma de adicionar esses fatores de riscos, adaptando assim à realidade dos clientes internados em CTI. Com isso otimizamos a perspectiva de prevenir a incidência de UP, reduzindo custos, tempo de internação e o sofrimento físico e psicológico dos pacientes.
9. Jorge SA, Dantas SRPE. Abordagem multiprofissional do tratamento de feridas. São Paulo: Atheneu; 2003.

10. Smeltzer SC, Bare BG. Tratado de enfermagem médico-cirúrgica. $9^{a}$ ed. Rio de Janeiro: Guanabarra Koogan; 2000.

11. Júnior Andrade J, Sales L. Sepse Brasil: estudo epidemiológico da Sepse em unidade de terapia intensiva brasileiras. Rev Bras Ter Int. 2006;18(1):9-17. 12. Ribeiro AG. A pele. In: Ribeiro AG, Sardenberg LM, Sandenberg JAGN. Tratamento de feridas. Goiânia: AB Cultura e Qualidade; 2004. p. 27-31. 13. Oliveira GMM. Influência da noradrenalina na mortalidade dos idosos com choque séptico. Rev SOCERJ. 2005;18(3):254-60.

14. Zeitoun SS. Incidência de pneumonia associada à ventilação mecânica em pacientes submetidos à aspiração endotraqueal pelos sistemas aberto e fechado: estudo prospectivo - dados preliminares. Rev Latinoam Enferm. 2001;9(1):46-52. 15. Fernandes LM. Úlceras de pressão em pacientes críticos hospitalizados. Uma revisão integrativa da literatura [dissertação]. Ribeirão Preto: Escola de Enfermagem de Ribeirão Preto da USP; 2000.

16. Hess CT. Tratamento de feridas e úlceras. Rio de janeiro: Reichmann \& Affonso; 2002.

17. Cunha NA. Sistematização da assistência de enfermagem no tratamento de feridas crônicas. Olinda: ABEn/PE; 2006.

18. Borges EL. Feridas: como tratar. Belo Horizonte: Coopmed Ed. Médica; 2001. 